\title{
Razaxaban Hydrochloride
}

National Cancer Institute

\section{Source}

National Cancer Institute. Razaxaban Hydrochloride. NCI Thesaurus. Code C81464.

The hydrochloride salt form of razaxaban, an orally active, 1,2-benzisoxazole-containing, inhibitor of coagulation factor Xa (activated factor X) with anticoagulant activity.

Razaxaban forms an isoxazole-ring opened, stable, benzamidine metabolite which is subsequently excreted through the bile. 